\title{
Estimation of Population Mean in Two Stage Design using Double Sampling for Stratification and Multiauxiliary Information
}

\author{
Monika Saini \\ Research Scholar Department of Statistics \\ M.D. University, Rohtak
}

\author{
Shashi Bahl \\ Prof. \& Head Department of Statistics \\ M.D. University, Rohtak
}

\begin{abstract}
For estimating the population mean of the heterogeneous population, when sampling frame in strata and the strata weight are not defined, we have proposed new difference and ratio estimators using double sampling for stratification and auxiliary information in two stage design. The expression for bias and variances of the proposed estimators have been obtained based on double sampling for stratification approach and comparison have been made with estimator under unstratified double sampling. In addition, we support these theoretical results by an empirical study.
\end{abstract}

\section{General Terms}

Sampling Theory

\section{Keywords}

Double Sampling for Stratification; Two Stage Design; Auxiliary Information; Difference (D) and Ratio (R) estimators.

\section{INTRODUCTION}

Information on variables correlated with the main variable under study is popularly known as auxiliary information which may be fruitfully utilized either at planning stage or at designing stage or at the estimation stage to arrive at improved estimator compared to those, not utilizing auxiliary information. If the required information is not available in advance for the entire population, it might be advantageous to collect certain items for large preliminary sample and then use this information at the estimation stage to improve the estimators. This technique is generally referred to as double sampling.

Stratification is one of the most widely used techniques in sample survey design serving the dual purpose of providing samples that are representative of major sub-groups of the population and improving the precision of estimators. Stratified sampling presupposes the knowledge of strata size as well as the availability of a frame for drawing a sample in each stratum. However application of this technique presupposes the knowledge of strata size and the availability of sampling frames within strata. But in many practical situations, when we want to estimate the population mean $\overline{\boldsymbol{Y}}$ of a variate $y$ and consider it desirable to stratify the population, consisting of $\mathrm{N}$ units on the basis of the value of auxiliary character $\mathrm{x}$ but the information of $\mathrm{x}$ is unknown, the sampling frame for various strata and the strata weight $\boldsymbol{W}_{\boldsymbol{h}}=\left(\boldsymbol{N}_{\boldsymbol{h}} \mid \boldsymbol{N}\right)(\mathrm{h}=1,2, \ldots \ldots \mathrm{L})$ are not known although the strata may be fixed in advance. In such situation the method of double sampling for stratification (DSS) can be used. Many authors including, Rao [3], Tripathi [7], Sukhatme et. al. [6]
Ige and Tripathi [1], Tripathi and Bahl [8], Singh and Vishwakarma [5] and Shabbir and Gupta [4] contributed well in the field of stratification.

In this paper we propose difference and ratio estimators in two stage design using DSS and multi-auxiliary information.

\section{SUGGESTED PROCEDURE}

Let a finite (survey) population $\mathrm{U}$ be partitioned into $\mathrm{N}$ fsu (first stage units) denoted by $\left(U_{1}, . ., U_{i}, . ., U_{N}\right)$ such that the number of ssu (second stage units) in $U_{i}$ be $M_{i}$. Suppose we want to estimate the population mean $\bar{Y}$ of study variate y and consider it desirable to stratify the population consisting of $\mathrm{N}$ units on the basis of one of values of the auxiliary characters $x_{k}^{\prime s}(\mathrm{k}=1,2, \ldots, \mathrm{p})$ but the frequency distribution of $x_{k}^{\prime s}$ is unknown. The sampling frame for various strata and the strata weight $\mathrm{W}_{\mathrm{h}}=\left(N_{h} \mid N\right),(\mathrm{h}=1,2 \ldots \mathrm{L})$, are not known although the strata may be fixed in advance. We use the technique of double sampling for stratification (DSS) in two stage design at fsu level; the sampling procedure consists of the following steps:

Step (1): We select a preliminary large sample of $n^{\prime}$ fsus rather inexpensively from a population of $\mathrm{N}$ fsus with simple random sampling without replacement and observe the auxiliary character $x_{1}, x_{2}, \ldots \ldots, x_{p}$ and then classify sample $n^{\prime}$ into L strata on the basis of the information obtained through $n^{\prime}$ for one or more $x_{k}^{\prime s}$. Let $n_{h}^{\prime}$ denote the number of units falling into stratum $\mathrm{h}(\mathrm{h}=1,2, \ldots ., \mathrm{L})$ i.e. $n_{1}^{\prime}, n_{2}^{\prime} \ldots \ldots . . n_{L}^{\prime}$ respectively, where $\sum_{h}^{L} n_{h}^{\prime}=n^{\prime}$.

Step (2): Randomly select $n_{h}$ i.e no. of fsu's in the sample, in stratum $\mathrm{h}$ out of $n_{h}^{\prime}$ without replacement, where $n_{h}=v_{h} n_{h}^{\prime}, 0<v_{h}<1(\mathrm{~h}=1,2 \ldots . \mathrm{L}) v_{h}$ being predetermined for each $\mathrm{h}$ are selected from strata independently and $\mathrm{n}=\sum n_{h}$; $n=\left(n_{1}, \ldots . . n_{L}\right)$.

Step (3): Randomly select $m_{h i}$ i.e no. of ssu`s to be selected from each sampled first stage units out of $M_{h i}$ in stratum h without replacement $(\mathrm{h}=1,2, \ldots ., \mathrm{L})$ and character of main interest $\mathrm{y}$ is observed.

Using above sampling scheme we propose difference and ratio estimators for the estimation of population mean.

\section{PROPOSED ESTIMATORS AND THEIR PROPERTIES}


Utilizing the information collected on $x_{1}, x_{2}, \ldots \ldots, x_{p}$, through the preliminary large sample $n^{\prime}$ on $x_{k}$-variates at fsu. We define multivariate difference and ratio estimators in two stage design using DSS at fsu level for the estimation of population mean and let us denote that estimator by $T_{D S S}$ for difference and ratio estimators i.e. $\left(T_{D S S}\right)_{D}$ and $\left(T_{D S S}\right)_{R}$ as

$$
\begin{aligned}
T_{D S S}=\sum a_{k} t_{k} \\
\text { as } t_{k}=\left\{\begin{array}{l}
\left(t_{k}\right)_{D}=\bar{y}_{(3)}-\lambda_{k}\left(\bar{x}_{k(2)}-\bar{x}_{k(1)}\right) \\
\left(t_{k}\right)_{R}=\left(\frac{\bar{y}_{(3)}}{\bar{x}_{k(2)}}\right) \bar{x}_{k(1)}
\end{array}\right.
\end{aligned}
$$

where $a=a_{1}, a_{2}, \ldots ., a_{p}$, with $\sum_{k}^{p} a_{k}=1$ is a weighting function and $\lambda_{k}^{\prime}$ s are suitably chosen constants.

In proposed estimator we note that

$$
\begin{aligned}
\bar{y}_{(3)} & =\sum_{h}^{L} w_{h}^{\prime} \frac{1}{n_{h}} \sum_{i}^{n_{h}} M_{h i} \bar{y}_{h i} \\
\bar{x}_{k(2)} & =\sum_{h}^{L} w_{h}^{\prime} \bar{x}_{k h} \\
\bar{x}_{k(1)} & =\sum_{h}^{L} w_{h}^{\prime} \bar{x}_{k h}^{\prime} \\
\text { such that } \quad \bar{y}_{h i} & =\frac{1}{m_{h i}} \sum_{j}^{m_{h i}} y_{h i j} \\
\bar{x}_{k h} & =\frac{1}{n_{h}} \sum_{i}^{n_{h}} x_{k h i} \\
\bar{x}_{k h}^{\prime} & =\frac{1}{n_{h}^{\prime}} \sum_{i}^{n_{h}^{\prime}} x_{k h i}
\end{aligned}
$$

where $\bar{y}_{(3)}, \bar{x}_{k(2)}$ and $\bar{x}_{k(1)}$ are unbiased estimator of population mean $\bar{Y}$ and $\bar{X}_{k}$. Note that $w_{h}^{\prime}=\frac{n_{h}^{\prime}}{n^{\prime}}$ is an unbiased estimator of strata weights $W_{h}=\frac{N_{h}}{N}$. Throughout we assume that $n^{\prime}$ is large enough so that $\operatorname{Pr}\left(n_{h}^{\prime}=0\right)=0$ for all $\mathrm{h}$. These estimators can shown to be unbiased by taking conditional expectation as

$$
\begin{aligned}
& E\left(\bar{y}_{(3)}\right)=E_{1} E_{2} E_{3}\left(\bar{y}_{(3)}\right)=\bar{Y} \\
& E\left(\bar{x}_{k(2)}\right)=E_{1} E_{2}\left(\bar{x}_{k(2)}\right)=\bar{X}_{k} \\
& E\left(\bar{x}_{k(1)}\right)=E_{1}\left(\bar{x}_{k(1)}\right)=\bar{X}_{k} \\
& \text { i.e. } \quad \bar{x}_{k(1)}=E_{2}\left(\bar{x}_{k(2)}\right)
\end{aligned}
$$

Theorem (3.1): The multivariate difference estimator $T_{D S S}$ is unbiased for $\bar{Y}$ and the exact expression for its variance is given by

$$
\begin{aligned}
V\left(T_{D S S}\right)_{D}=\left(\frac{1}{n^{\prime}}-\frac{1}{N}\right) S_{y}^{2} & +\frac{1}{n^{\prime}} \sum_{h} W_{h}\left(\frac{1}{v_{h}}-1\right) a^{\prime} B_{h} a \\
& +\frac{1}{N} \sum_{h} \frac{W_{h}}{n_{h}} \sum_{i} M_{h i}^{2}\left(\frac{1}{m_{h i}}-\frac{1}{M_{h i}}\right) S_{h y i}^{2}
\end{aligned}
$$

with $B_{h}=\left(b_{h k l}\right) \quad \mathrm{h}=1,2, \ldots \ldots ., \mathrm{L} \quad ; \quad \mathrm{k}, \mathrm{l}=1,2, \ldots \ldots, \mathrm{p}$

$$
\left(b_{h k l}\right)=\left(S_{h y}^{2}-\lambda_{k} S_{h y k}-\lambda_{l} S_{h y l}-\lambda_{k} \lambda_{l} S_{h k l}\right)
$$

Further for large samples, the approximate expression for the bias and mean square error of $\left(T_{D S S}\right)_{R}$ is given by

$$
B\left(T_{D S S}\right)_{R}=\frac{1}{n^{\prime}} \sum_{k} \frac{a_{k}}{\bar{X}_{k}} \sum_{h} W_{h}\left(\frac{1}{v_{h}}-1\right)\left(R_{k} S_{h k}^{2}-S_{h y k}\right)
$$

$$
\begin{aligned}
& \operatorname{MSE}\left(T_{D S S}\right)_{R}=V\left(T_{D S S}\right)_{D} \quad \lambda_{k}=R_{k} \\
& \lambda_{l}=R_{l} \quad(\mathrm{k}, 1=1,2, \ldots \ldots, \mathrm{p})
\end{aligned}
$$

Proof: Since $\quad T_{D S S}=\sum a_{k} t_{k}$

$$
\begin{aligned}
& \left(t_{k}\right)_{D}=\bar{y}_{(3)}-\lambda_{k}\left(\bar{x}_{k(2)}-\bar{x}_{k(1)}\right) \\
& E\left(T_{D S S}\right)_{D}=E_{1} E_{2} E_{3}\left(T_{D S S}\right)_{D} \\
& E_{2} E_{3}\left(t_{k}\right)=\bar{y}^{\prime} \\
& E\left(T_{D S S}\right)_{D}=E_{1} E_{2} E_{3}\left(T_{D S S}\right)_{D}=\bar{Y}
\end{aligned}
$$

The multivariate difference estimator $T_{D S S}$ for $\bar{Y}$ is unbiased. The exact expression for its variance is given as

$$
\begin{aligned}
& \mathrm{V}\left(\mathrm{T}_{\mathrm{DSS}}\right)_{\mathrm{D}}=\sum_{\mathrm{k}} \sum_{\mathrm{l}} \mathrm{a}_{\mathrm{k}} \mathrm{a}_{\mathrm{l}} \operatorname{Cov}\left(\mathrm{t}_{\mathrm{k}}, \mathrm{t}_{\mathrm{l}}\right) \\
& \operatorname{Cov}\left(\mathrm{t}_{\mathrm{k}} \mathrm{t}_{\mathrm{l}}\right)=\mathrm{v}\left(\overline{\mathrm{y}}_{(3)}\right)-\lambda_{\mathrm{l}} \operatorname{Cov}\left(\overline{\mathrm{y}}_{(3)}, \overline{\mathrm{x}}_{\mathrm{l}(2)}\right) \\
& +\lambda_{\mathrm{k}} \operatorname{Cov}\left(\overline{\mathrm{y}}_{(3)}, \overline{\mathrm{x}}_{\mathrm{k}(1)}\right)-\lambda_{\mathrm{k}} \operatorname{Cov}\left(\overline{\mathrm{y}}_{(3)}, \overline{\mathrm{x}}_{\mathrm{k}(2)}\right) \\
& +\lambda_{\mathrm{l}} \operatorname{Cov}\left(\overline{\mathrm{y}}_{(3)}, \overline{\mathrm{x}}_{\mathrm{l}(1)}\right)+\lambda_{\mathrm{k}} \lambda_{\mathrm{l}}\left[\operatorname { C o v } \left(\overline{\mathrm{x}}_{\mathrm{k}(2)}, \overline{\mathrm{x}}_{\mathrm{l}(2)}\right.\right. \\
& -\operatorname{Cov}\left(\overline{\mathrm{x}}_{\mathrm{k}(2)}, \overline{\mathrm{x}}_{\mathrm{l}(1)}\right)-\operatorname{Cov}\left(\overline{\mathrm{x}}_{\mathrm{k}(1)}, \overline{\mathrm{x}}_{\mathrm{l}(2)}\right)+\operatorname{Cov}\left(\overline{\mathrm{x}}_{\mathrm{k}(1)}, \overline{\mathrm{x}}_{\mathrm{l}(1)}\right) \\
& V\left(T_{D S S}\right)_{D}=\left(\frac{1}{n^{\prime}}-\frac{1}{N}\right) S_{y}^{2}+\frac{1}{n^{\prime}} \sum_{h} W_{h}\left(\frac{1}{v_{h}}-1\right) a^{\prime} B_{h} a \\
& +\frac{1}{N} \sum_{h} \frac{W_{h}}{n_{h}} \sum_{i} M_{h i}^{2}\left(\frac{1}{m_{h i}}-\frac{1}{M_{h i}}\right) S_{i h y}^{2} \\
& \left(b_{h k l}\right)=\left(S_{h y}^{2}-\lambda_{k} S_{h y k}-\lambda_{l} S_{h y l}-\lambda_{k} \lambda_{l} S_{h k l}\right) \\
& S_{h y i}^{2}=\frac{1}{M_{h i-1}} \sum_{j}^{M_{i h}}\left(y_{h i j}-\bar{Y}_{i h}\right)^{2}
\end{aligned}
$$

To obtain the bias and mean square error of multivariate ratio estimator $\left(T_{D S S}\right)_{R}$ we use the delta notation

$$
\begin{aligned}
& e_{o}=\frac{\bar{y}_{(3)}-\bar{Y}}{\bar{Y}} \\
& e_{1}=\frac{\bar{x}_{k(2)}-\bar{X}_{k}}{\bar{X}_{k}} \\
& e_{2}=\frac{\bar{x}_{k(1)}-\bar{X}_{k}}{\bar{X}_{k}} \\
& \bar{y}_{(3)}=\bar{Y}\left[1+e_{o}\right] \\
& \bar{x}_{k(2)}=\bar{X}_{k}\left[1+e_{1}\right] \quad \bar{x}_{k(1)}=\bar{X}_{k}\left[1+e_{2}\right]
\end{aligned}
$$

now equation (3.3) becomes

$\left(T_{D S S}\right)_{R}=\sum a_{k} \bar{Y}\left[1+e_{o}\right]\left[1+e_{1}\right]^{-1}\left[1+e_{2}\right]$

Further for large samples, neglecting terms of higher powers, the approximate expression for its bias and mean square error is given by 


$$
\begin{gathered}
B\left(T_{D S S}\right)_{R}=\frac{1}{n^{\prime}} \sum_{k} \frac{a_{k}}{\bar{X}_{k}} \sum_{h} W_{h}\left(\frac{1}{v_{h}}-1\right)\left(R_{k} S_{h k}^{2}-S_{h y k}\right) \\
M\left(T_{D S S}\right)_{R}=\left(\frac{1}{n^{\prime}}-\frac{1}{N}\right) S_{y}^{2}+\frac{1}{n^{\prime}} \sum_{h} W_{h}\left(\frac{1}{v_{h}}-1\right) a^{\prime} B_{h} a \\
+\frac{1}{N} \sum_{h} \frac{W_{h}}{n_{h}} \sum_{i} M_{h i}^{2}\left(\frac{1}{m_{h i}}-\frac{1}{M_{h i}}\right) S_{i h y}^{2}
\end{gathered}
$$

with $\quad B_{h}=\left(b_{h k l}\right) \quad \mathrm{h}=1,2 \ldots, \mathrm{L} ; \quad \mathrm{k}, \mathrm{l}=1,2, \ldots \ldots \mathrm{p}$

$$
\left(b_{\text {hkl }}\right)=\left(S_{\text {hy }}^{2}-R_{k} S_{\text {hyk }}-R_{l} S_{\text {hyl }}-R_{k} R_{l} S_{\text {hkl }}\right)
$$

where $\quad \mathrm{S}_{\mathrm{hkl}}=\frac{1}{\mathrm{~N}_{\mathrm{h}-1}} \sum_{\mathrm{i}}^{\mathrm{N}_{\mathrm{h}}}\left(\mathrm{x}_{\mathrm{hki}}-\overline{\mathrm{X}}_{\mathrm{hk}}\right)\left(\mathrm{x}_{\mathrm{hli}}-\overline{\mathrm{X}}_{\mathrm{hl}}\right)$

$$
\mathrm{S}_{\text {hyi }}^{2}=\frac{1}{\mathrm{M}_{\mathrm{hi}-1}} \sum_{j}^{M_{i h}}\left(y_{h i j}-\bar{Y}_{i h}\right)^{2}
$$

Remark: From equation (3.4) and (3.5) is can easily be proved that for large sample

$$
\begin{aligned}
& \mathrm{M}\left(\mathrm{T}_{\mathrm{DSS}}\right)_{\mathrm{R}}=\mathrm{V}\left(\mathrm{T}_{\mathrm{DSS}}\right)_{\mathrm{D}} \quad \text { for } \quad \lambda_{\mathrm{k}}=\mathrm{R}_{\mathrm{k}} \\
& \lambda_{\mathrm{l}}=\mathrm{R}_{\mathrm{l}}
\end{aligned}
$$

\section{OPTIMUM ESTIMATORS}

Let $\beta_{\mathrm{ok}}=\sum_{\mathrm{h}} \mathrm{c}_{\mathrm{kh}} \beta_{\mathrm{okh}} / \sum_{\mathrm{h}} \mathrm{c}_{\mathrm{kh}}$ with $\mathrm{c}_{\mathrm{kh}}=\mathrm{W}_{\mathrm{h}}\left(\frac{1}{\mathrm{v}_{\mathrm{h}}}-1\right) \mathrm{S}_{\mathrm{hk}}^{2}$ be the weighted average of the strata population regression coefficients $\beta_{\mathrm{okh}}=\mathrm{S}_{\mathrm{hyk}} / \mathrm{S}^{2}{ }_{\mathrm{hk}}$ of $\mathrm{y}$ on $\mathrm{x}_{\mathrm{k}}$ and

$\rho_{\mathrm{kl}}=\frac{\sum_{\mathrm{h}} \mathrm{W}_{\mathrm{h}}\left(\frac{1}{v_{\mathrm{h}}}-1\right) \rho_{\mathrm{hkl}} \mathrm{S}_{\mathrm{hk}} \mathrm{S}_{\mathrm{hl}}}{\left[\sum_{\mathrm{h}} \mathrm{W}_{\mathrm{h}}\left(\frac{1}{v_{\mathrm{h}}}-1\right) \mathrm{S}_{\mathrm{hk}}^{2} \sum_{\mathrm{h}} \mathrm{W}_{\mathrm{h}}\left(\frac{1}{v_{\mathrm{h}}}-1\right) \mathrm{S}_{\mathrm{hl}}^{2}\right]^{1 / 2}}$

where $\rho_{\mathrm{hkl}}=\mathrm{S}_{\mathrm{hkl}} / \mathrm{S}_{\mathrm{hk}} \mathrm{S}_{\mathrm{hl}}$ is the correlation coefficient between $x_{k}$ and $x_{l}$ in stratum $h$.

For $\mathrm{p}=1$, when information on only $\mathrm{x}_{\mathrm{k}}$, is used, following Ige and Tripathi [1] the optimum value of $\lambda_{k}$ is given by

$$
\lambda_{\mathrm{ok}}=\frac{\sum_{\mathrm{h}} \mathrm{W}_{\mathrm{h}}\left(\frac{1}{\mathrm{v}_{\mathrm{h}}}-1\right) \mathrm{S}_{\mathrm{hyk}}}{\sum_{\mathrm{h}} \mathrm{W}_{\mathrm{h}}\left(\frac{1}{\mathrm{v}_{\mathrm{h}}}-1\right) \mathrm{S}_{\mathrm{hk}}^{2}}=\beta_{\mathrm{ok}}
$$

When the choices for each $\lambda_{\mathrm{k}}=\beta_{\mathrm{ok}}$ are made for each $\mathrm{k}$, the resulting variance is given by

$$
\begin{aligned}
\mathrm{V}\left(\mathrm{T}_{\mathrm{DSS}}\right)_{\lambda_{\mathrm{k}}=\beta_{\mathrm{ok}}}=\left(\frac{1}{\mathrm{n}^{\prime}}-\frac{1}{\mathrm{~N}}\right) \mathrm{S}_{\mathrm{y}}^{2} \\
+\frac{1}{\mathrm{n}^{\prime}} \sum_{\mathrm{h}} \mathrm{W}_{\mathrm{h}}\left(\frac{1}{\mathrm{v}_{\mathrm{h}}}-1\right) \mathrm{S}_{\mathrm{hy}}^{2}\left(1-\rho_{\mathrm{yk}}^{2}-\rho_{\mathrm{yl}}^{2}+\rho_{\mathrm{yk}} \rho_{\mathrm{yl}} \rho_{\mathrm{kl}}\right) \\
+\frac{1}{\mathrm{~N}} \sum_{\mathrm{h}} \frac{\mathrm{W}_{\mathrm{h}}}{\mathrm{n}_{\mathrm{h}}} \sum_{\mathrm{i}} \mathrm{M}_{\mathrm{hi}}^{2}\left(\frac{1}{\mathrm{~m}_{\mathrm{hi}}}-\frac{1}{\mathrm{M}_{\mathrm{hi}}}\right) \mathrm{S}_{\mathrm{yhi}}^{2}
\end{aligned}
$$

Further, when optimum weight vector

$$
\mathrm{a}_{\mathrm{o}}=\frac{\mathrm{B}^{-1} \mathrm{~g}}{\mathrm{~g}^{\prime} \mathrm{B}^{-1} \mathrm{~g}} \quad \mathrm{~g}=(1,1, \ldots, 1)^{\prime}
$$

is used, we obtain

$$
\begin{aligned}
\mathrm{V}\left(\mathrm{T}_{\mathrm{DSS}}\right)_{\substack{\lambda_{\mathrm{k}}=\beta_{\mathrm{ok}} \\
\mathrm{a}=\mathrm{a}_{\mathrm{o}}}}=\left(\frac{1}{\mathrm{n}^{\prime}}-\frac{1}{\mathrm{~N}}\right) \mathrm{S}_{\mathrm{y}}^{2} \\
+\frac{1}{\mathrm{n}^{\prime}}\left(\mathrm{g}^{\prime} \mathrm{B}^{-1} \mathrm{~g}\right)^{-1} \sum_{\mathrm{h}} \mathrm{W}_{\mathrm{h}}\left(\frac{1}{\mathrm{v}_{\mathrm{h}}}-1\right) \mathrm{S}_{\mathrm{hy}}^{2}
\end{aligned}
$$

$$
+\frac{1}{\mathrm{n}^{\prime}} \sum_{\mathrm{h}} \frac{\mathrm{W}_{\mathrm{h}}}{\mathrm{n}_{\mathrm{h}}} \sum_{\mathrm{i}} \mathrm{M}_{\mathrm{hi}}^{2}\left(\frac{1}{\mathrm{~m}_{\mathrm{hi}}}-\frac{1}{\mathrm{M}_{\mathrm{hi}}}\right) \mathrm{S}_{\mathrm{yhi}}^{2}
$$

In practice, when exact value of $\lambda_{\mathrm{ok}}=\beta_{\mathrm{ok}}$ is not available, it may be estimated through the sample data

$$
\hat{\beta}_{\mathrm{ok}}=\frac{\sum_{\mathrm{h}} \mathrm{w}_{\mathrm{h}}^{\prime}\left(\frac{1}{v_{\mathrm{h}}}-1\right) \mathrm{s}_{\mathrm{hyk}}}{\sum_{\mathrm{h}} \mathrm{w}_{\mathrm{h}}^{\prime}\left(\frac{1}{v_{\mathrm{h}}}-1\right) \mathrm{s}_{\mathrm{hk}}^{2}}
$$

where

$$
\begin{aligned}
s_{\text {hyk }} & =\frac{1}{n_{h}-1} \sum_{i}^{n_{h}}\left(y_{i h}-\bar{y}_{h}\right)\left(x_{h k i}-\bar{x}_{k h}\right) \\
s_{h k}^{2} & =s_{h k k}
\end{aligned}
$$

are unbiased estimator for $S_{\text {hyk }}$ and $S_{\text {hk }}^{2}$ respectively.

Using the estimated optimum values, we may define a multiple regression estimator for $\bar{Y}$ in DSS by

$$
\left(\mathrm{T}_{\mathrm{DSS}}\right)_{\text {reg }}^{1}=\overline{\mathrm{y}}_{(3)}-\sum_{\mathrm{k}} \mathrm{a}_{\mathrm{k}} \hat{\beta}_{\mathrm{ok}}\left(\overline{\mathrm{x}}_{\mathrm{k}(2)}-\overline{\mathrm{x}}_{\mathrm{k}(1)}\right)
$$

for large samples, $\mathrm{M}\left(\mathrm{T}_{\mathrm{DSS}}\right)_{\text {reg }}$ would again be given by (4.1).

One may in fact obtain simultaneous optimum value of $\mathrm{T}_{\mathrm{k}}=\mathrm{a}_{\mathrm{k}} \lambda_{\mathrm{k}}(\mathrm{k}=1,2, \ldots \ldots, \mathrm{p})$ as follows.

Let

$$
\mathrm{S}^{*}=\left(\mathrm{S}_{\mathrm{kl}}^{*}\right) \quad \mathrm{Q}=\left(\mathrm{Q}_{1}, \mathrm{Q}_{2}, \ldots \ldots, \mathrm{Q}_{\mathrm{p}}\right)^{\prime}
$$

where $\mathrm{S}_{\mathrm{kl}}^{*}=\sum_{\mathrm{h}} \mathrm{W}_{\mathrm{h}}\left(\frac{1}{\mathrm{v}_{\mathrm{h}}}-1\right) \mathrm{S}_{\mathrm{hkl}}$

$$
\mathrm{Q}_{\mathrm{k}}=\mathrm{S}_{\mathrm{yk}}^{*} \quad \mathrm{k}, \mathrm{l}=1,2, \ldots, \mathrm{p}
$$

then $V\left(T_{D S S}\right)=\left(\frac{1}{n^{\prime}}-\frac{1}{N}\right) S_{y}^{2}+\frac{1}{n^{\prime}}\left(S_{y}^{* 2}-2 T^{\prime} Q+T^{\prime} S^{*} T\right)$

$$
+\frac{1}{\mathrm{~N}} \sum_{\mathrm{h}} \frac{\mathrm{W}_{\mathrm{h}}}{\mathrm{n}_{\mathrm{h}}} \sum_{\mathrm{i}} \mathrm{M}_{\mathrm{hi}}^{2}\left(\frac{1}{\mathrm{~m}_{\mathrm{hi}}}-\frac{1}{\mathrm{M}_{\mathrm{hi}}}\right) \mathrm{S}_{\mathrm{yhi}}^{2}
$$

which gives $\quad \mathrm{T}_{\mathrm{opt}}=\mathrm{T}_{\mathrm{o}}=\mathrm{S}^{*-1} \mathrm{Q}$

$$
\begin{aligned}
\mathrm{V}\left(\mathrm{T}_{\text {DSS }}\right)_{\text {opt }}= & \left(\frac{1}{\mathrm{n}^{\prime}}-\frac{1}{\mathrm{~N}}\right) \mathrm{S}_{\mathrm{y}}^{2}+\frac{1}{\mathrm{n}^{\prime}} \mathrm{S}_{\mathrm{y}}^{* 2}\left(1-\mathrm{R}^{2}\right) \\
& +\frac{1}{\mathrm{~N}} \sum_{\mathrm{h}} \frac{\mathrm{W}_{\mathrm{h}}}{\mathrm{n}_{\mathrm{h}}} \sum_{\mathrm{i}} \mathrm{M}_{\mathrm{hi}}^{2}\left(\frac{1}{\mathrm{~m}_{\mathrm{hi}}}-\frac{1}{\mathrm{M}_{\mathrm{hi}}}\right) \mathrm{S}_{\mathrm{yhi}}^{2}
\end{aligned}
$$

where $\mathrm{R}^{2}=\frac{\mathrm{Q}^{\prime} \mathrm{S}^{*-1} \mathrm{Q}}{\mathrm{S}_{\mathrm{y}}^{* 2}}$ and $\mathrm{R}$ being the multiple is the correlation coefficient between $\bar{y}_{(3)}$ and $\left(\bar{x}_{\mathrm{k}(2)}-\overline{\mathrm{x}}_{\mathrm{k}(1)}\right)$.

The optimum value of $\mathrm{T}$ may be estimated by

$$
\widehat{T}=\widehat{\mathrm{S}}^{*-1} \widehat{\mathrm{Q}} \quad \widehat{\mathrm{S}}^{*}=\left(\widehat{\mathrm{S}}_{\mathrm{kl}}^{*}\right) \quad \widehat{\mathrm{Q}}=\left(\widehat{\mathrm{Q}}_{1}, \widehat{\mathrm{Q}}_{2}, \ldots \ldots, \widehat{\mathrm{Q}}_{\mathrm{p}}\right)^{\prime}
$$

where $\quad \widehat{\mathrm{S}}_{\mathrm{kl}}^{*}=\sum_{\mathrm{h}} \mathrm{w}_{\mathrm{h}}^{\prime}\left(\frac{1}{\mathrm{v}_{\mathrm{h}}}-1\right) \mathrm{s}_{\mathrm{hkl}} \quad \widehat{\mathrm{Q}}_{\mathrm{k}}=\widehat{\mathrm{S}}_{\mathrm{yk}}^{*}$

Using these estimated values, we may define a multiple regression estimator based on DSS for $\bar{Y}$ as

$$
\left(\mathrm{T}_{\mathrm{DSS}}\right)_{\text {reg }}=\overline{\mathrm{y}}_{(3)}-\sum_{\mathrm{k}} \widehat{\mathrm{T}}_{\mathrm{k}}^{*}\left(\overline{\mathrm{x}}_{\mathrm{k}(2)}-\overline{\mathrm{x}}_{\mathrm{k}(1)}\right)
$$

whose variance for large samples is given by (4.2). 


\section{COMPARISION}

In this section we compare the proposed multivariate difference and ratio estimators based on DSS with Prabha [2] in Unstratified Double Sampling (USDS) where second sample is a simple random at size $\mathrm{n}$ from the first random sample $n$ 'instead of being selected in the form of stratified sub-samples.

The multivariate difference and ratio estimator for the difference and ratio estimator for the population mean in USDS for the population mean in USDS are defined by:

$$
T=\sum_{k}^{p} a_{k} d_{k}
$$

where

$$
\begin{array}{ll}
\left(d_{k}\right)_{D}=\overline{\bar{y}}_{w}-\lambda_{k}\left(\bar{x}_{w}-\bar{x}^{\prime}{ }_{w}\right) & \\
\left(d_{\mathrm{k}}\right)_{R}=\frac{\bar{y}_{w}}{\bar{x}_{w}} \bar{x}_{w}^{\prime} & \mathrm{k}=1,2, \ldots \ldots, \mathrm{p}
\end{array}
$$

where the weights $a_{k}(\mathrm{k}=1,2, \ldots \mathrm{p})$ add ud to unity and $\lambda_{k}$ are suitably chosen constants with

$$
\begin{aligned}
V(T)_{D}=( & \left.\frac{1}{\mathrm{n}^{\prime}}-\frac{1}{\mathrm{~N}}\right) \mathrm{S}_{\mathrm{y}}^{2}+\left(\frac{1}{\mathrm{n}}-\frac{1}{\mathrm{n}^{\prime}}\right) \mathrm{a}^{\prime} \mathrm{Ba} \\
& +\frac{1}{n N} \sum_{i}^{N} M_{i}^{2}\left(\frac{1}{m_{i}}-\frac{1}{M_{i}}\right) S_{i y}^{2}
\end{aligned}
$$

with $\mathrm{B}=\left(b_{k l}\right)$

$$
\begin{aligned}
\left(b_{k l}\right)=\left(S_{y}^{2}-\right. & \left.\lambda_{k} S_{y k}-\lambda_{l} S_{y l}+\lambda_{k} \lambda_{l} S_{k l}\right) \\
M(T)_{R}=\left(\frac{1}{\mathrm{n}^{\prime}}-\frac{1}{\mathrm{~N}}\right) S_{\mathrm{y}}^{2} & +\left(\frac{1}{\mathrm{n}}-\frac{1}{\mathrm{n}^{\prime}}\right) \mathrm{a}^{\prime} \mathrm{Ba} \\
& +\frac{1}{n N} \sum_{i}^{N} M_{i}^{2}\left(\frac{1}{m_{i}}-\frac{1}{M_{i}}\right) S_{i y}^{2}
\end{aligned}
$$

with $\mathrm{B}=\left(b_{k l}\right)$

$$
\begin{aligned}
& \left(b_{k l}\right)=\left(S_{y}^{2}-R_{k} S_{y k}-R_{l} S_{y l}+R_{k} R_{l} S_{k l}\right) \\
& M(T)_{R}=V(T)_{D}
\end{aligned}
$$

We note that both of $\left(T_{D S S}\right)_{D}$ and $(\mathrm{T})_{D}$ are unbiased and both have exact expression for their variances for all sample sizes while $\left(T_{D S S}\right)_{R}$ and $(\mathrm{T})_{R}$ are biased and the expression for their MSE is approximated (for large samples). Further, the information on $x_{1}, x_{2}, \ldots, x_{p}$ is basic for all the above estimators, it is being used both for stratification as well as for constructing estimators in case of DSS while only for defining estimators in case of USDS.

Using

$$
\begin{aligned}
& S_{k l}=\sum_{h} W_{h} S_{h k l}+\sum_{h} W_{h}\left(\bar{x}_{k h}-\bar{X}_{k}\right)\left(\bar{x}_{l h}-\bar{X}_{l}\right) \\
& S_{y k}=\sum_{h} W_{h} S_{h y k}+\sum_{h} W_{h}\left(\bar{Y}_{h}-\bar{Y}\right)\left(\bar{x}_{k h}-\bar{X}_{k}\right) \\
& \text { we get } b_{k l}=\left(S_{y}^{2}-\lambda_{k} S_{y k}-\lambda_{l} S_{y l}+\lambda_{k} \lambda_{l} S_{k l}\right) \\
& \begin{array}{c}
=\sum_{h} W_{h}\left(S_{h y}^{2}-\lambda_{k} S_{h y k}-\lambda_{l} S_{h y l}+\lambda_{k} \lambda_{l} S_{h k l}\right) \\
+\sum_{h} W_{h}\left(\bar{Y}_{h}-\bar{Y}\right)^{2}-\lambda_{k}\left(\bar{Y}_{h}-\bar{Y}\right)\left(\bar{x}_{k h}-\bar{X}_{k}\right) \\
-\lambda_{l}\left(\bar{Y}_{h}-\bar{Y}\right)\left(\bar{x}_{l h}-\bar{X}_{l}\right)+\lambda_{k} \lambda_{l}\left(\bar{x}_{k h}-\bar{X}_{k}\right)\left(\bar{x}_{l h}-\bar{X}_{l}\right)
\end{array}
\end{aligned}
$$

For comparisons assuming

$$
\begin{aligned}
& \frac{n_{h}}{n_{h}^{\prime}}=\frac{n}{n^{\prime}}=v_{h} \\
& V\left(T_{D S S}\right)=\left(\frac{1}{n^{\prime}}-\frac{1}{N}\right) S_{y}^{2}+ \\
& \left(\frac{1}{n}-\frac{1}{n}\right) \sum \sum a_{k} a_{l} \sum_{h} W_{h}\left(S_{h y}^{2}-\lambda_{k} S_{h y k}-\lambda_{l} S_{h y l}+\lambda_{k} \lambda_{l} S_{h k l}\right) \\
& +\frac{1}{n^{\prime}} \sum_{h} \frac{W_{h}}{n_{h}} \sum_{i} M_{h i}^{2}\left(\frac{1}{m_{h i}}-\frac{1}{M_{h i}}\right) S_{h y i}^{2} \\
& \text { Then } V(T)_{D}-V\left(T_{D S S}\right)_{D}=\left(\frac{1}{n}-\frac{1}{n}\right) \sum \sum a_{k} a_{l} \sum_{h} W_{h} d_{h k l}^{(1)} \\
& \text { where } d_{h k l}^{(1)}=\left(\bar{Y}_{h}-\bar{Y}\right)^{2}-\lambda_{k}\left(\bar{Y}_{h}-\bar{Y}\right)\left(\bar{x}_{k h}-\bar{X}_{k}\right) \\
& -\lambda_{l}\left(\bar{Y}_{h}-\bar{Y}\right)\left(\bar{x}_{l h}-\bar{X}_{l}\right)+\lambda_{k} \lambda_{l}\left(\bar{x}_{k h}-\bar{X}_{k}\right)\left(\bar{x}_{l h}-\bar{X}_{l}\right) \\
& =\left(\left(\bar{Y}_{h}-\bar{Y}\right)-\lambda_{k}\left(\bar{x}_{k h}-\bar{X}_{k}\right)\right)\left(\left(\bar{Y}_{h}-\bar{Y}\right)-\lambda_{l}\left(\bar{x}_{l h}-\bar{X}_{l}\right)\right) \\
& =\left(\frac{1}{n}-\frac{1}{n^{\prime}}\right) \sum_{h} W_{h} a^{\prime} D_{h}^{(1)} a \\
& D_{h}^{(1)}=d_{h k l}^{(1)} \\
& M(T)_{R}-M\left(T_{D S S}\right)_{R}=\left(\frac{1}{n}-\frac{1}{n}\right) \sum \sum a_{k} a_{l} \sum_{h} W_{h} d_{h k l}^{(2)} \\
& D_{h}^{(2)}=d_{h k l}^{(2)} \\
& d_{h k l}^{(2)}=\left[( \overline { Y } _ { h } - R _ { k } \overline { X } _ { k h } ] \left[\left(\bar{Y}_{h}-R_{l} \bar{X}_{l h}\right] \quad k, l=1,2, \ldots \ldots, p\right.\right.
\end{aligned}
$$

It is to be noted that $D_{h}^{(1)}$ and $D_{h}^{(2)}$ are positive definite matrices which proves that under proportional allocation of the second sample, the multivariate difference and ratio estimators in DSS are always better than corresponding estimators in UDSS

\section{EMPIRICAL STUDY}

To show the usefulness of the proposed estimators based on DSS compared to estimators based on UDSS suggested by Prabha (1992) in two stage design. It can be checked by comparing their variances and percent relative efficiency, numerically, an empirical study has been carried out.

Population: For this we consider the 2001 census data which is relates to the total number of agricultural labourers, total area, total population, and total no. of cultivators of 444 villages of Bhiwani district of Haryana. We take number of agricultural labourers in villages as $y$,the total area of villages as $x_{1}$, the total population of villages as $x_{2}$, and the total no. of cultivators as $x_{3}$. The whole population of Bhiwani district (444 villages) is divided into villages at fsu level and household at ssu level.

The whole population of 444 village's fsus is stratified into three strata according to area. So the strata become as under:

\begin{tabular}{|l|l|}
\hline Strata & Area in Hectare \\
\hline I & $1-800 \quad(227$ villages $)$ \\
\hline II & $800-1660(135$ villages $)$ \\
\hline III & $1660-2400 \&$ above $(82$ villages $)$ \\
\hline
\end{tabular}

The numerical values of the estimate of the population mean,its variances are worked out under each of the proposed estimators from population values. Subsequently, the percent 
relative efficiency of each of the estimator given in this paper is calculated. The result are presented in the form of Table II given below

Table I

\section{Description of Population}

\begin{tabular}{|l|l|}
\hline Source & $\begin{array}{c}\text { Village wise information of Bhiwani District } \\
\text { of Haryana (2001 census data) }\end{array}$ \\
\hline $\mathrm{y}$ & study variable ( agricultural laborers) \\
\hline$x_{1}$ & auxiliary information (total area) \\
\hline$x_{2}$ & auxiliary information (total population) \\
\hline$x_{3}$ & auxiliary information (total number of cultivators) \\
\hline
\end{tabular}

\section{Table II}

\begin{tabular}{|c|l|l|l|}
\hline (i) & \multicolumn{1}{|c|}{ (ii) } & (iii) & (iv) \\
\hline Estimators & $\begin{array}{c}\text { Auxiliary } \\
\text { variable used } \\
\times \mathbf{1 0}^{\mathbf{7}}\end{array}$ & $\begin{array}{l}\text { Rariance } \% \\
\text { w.r.t. }(\mathbf{T})_{\boldsymbol{D}}\end{array}$ \\
\hline$(T)_{D}$ & $x_{1}, x_{2}$ and $x_{3}$ & 2.27 & 100 \\
$\left(T_{D S S}\right)_{D}$ & $x_{1}, x_{2}$ and $x_{3}$ & 1.78 & 127 \\
\hline & & 2.24 & 100 \\
$(T)_{R}$ & $x_{1}, x_{2}$ and $x_{3}$ & 1.72 & 130 \\
$\left(T_{D S S}\right)_{R}$ & $x_{1}, x_{2}$ and $x_{3}$ & R.r.t. $(\mathbf{T})_{\boldsymbol{R}}$ \\
\hline
\end{tabular}

\section{CONCLUSION}

From table II, column III, we interpret that the estimators based on DSS give less variance as compare to USDS and in column IV estimators based on DSS have more relative efficiency as compare to USDS.

From the perusal of above table we conclude that estimators based on DSS at fsu level in two stage design as compare to the estimators based on USDS. Thus the estimator $\left(T_{D S S}\right)_{D}$ and $\left(T_{D S S}\right)_{R}$ are recommended for used in practice for the estimation of population mean

\section{REFERENCES}

[1] Ige, A.F. and Tripathi, T.P. 1987. On double sampling for stratification and use of auxiliary information. Jour. Ind. Soc. Ag. Stat. 39, 191-201.

[2] Prabha, S. 1992. On Some Estimation Problems in Sampling Using Multiauxiliary Information. Ph.D Thesis, M.D. University, Rohtak

[3] Rao, J.N.K. 1973. On double sampling for stratification and analytical surveys. Biometrika 60, 125-133.

[4] Shabbir, J. and Gupta S. 2011. On Estimating Finite Population Mean in Simple and Stratified Random Sampling. Commun. in Stat. 40, 199-212.

[5] Singh, H.P. and Vishwakarma, G.K. 2010. A general procedure for estimating the population mean in Stratified sampling using auxiliary information, Metron, vol LXVIII, no.1, 47-65.

[6] Sukhatme, P. V., Sukhatme, B.V. Sukhatme, S. and Ashok, C. 1984.Sampling Theory of Surveys with Applications, Iowa State University Press, Ames, Iowa.

[7] Tripathi, T.P. 1976. On double sampling for multi-variate ratio and difference methods of estimation. Jour. Ind. Soc. Agr. Stat. 38, 33-34.

[8] Tripathi, T. P. and Bahl, S. 1991. Estimation of mean using double Sampling for Stratification and Multi auxiliary Information, Common. Statist. Theory Meth. 20, 8, 2589-2602. 